\title{
The direct recovery of recombinant hepatitis $B$ core antigen from disruptate derived from continuous-flow bead milling.
}

\begin{abstract}
$\mathrm{HBcAg}$ (hepatitis B core antigen) is a nanoplex bioproduct that has a great potential in the development of therapeutic drugs and vaccines. In the present study, a continuous-flow bead milling for the disruption of Escherichia coli was optimized and a direct recovery protocol to isolate the recombinant $\mathrm{HBcAg}$ from the unclarified $\mathrm{E}$. coli disruptate was developed. The optimal condition for continuous-flow bead milling for the release of $\mathrm{HBcAg}$ from E. coli was achieved at a feed flow rate of 15 litres/h, biomass concentration of $10 \%$ [ww/v (wet weight/vol.)] and impeller tip speed of $14 \mathrm{~m} / \mathrm{s}$. The sucrose-density-gradient analysis showed that the particulate form of the $\mathrm{HBcAg}$ released by this optimal condition is still preserved. In the direct purification of $\mathrm{HBcAg}$ from the unclarified disruptate, the AE-EBAC (anionexchange expanded-bed adsorption chromatography) technique was employed. A 54\% adsorption and $50.7 \%$ recovery of $\mathrm{HBcAg}$ were achieved in this direct recovery process. The purity of $\mathrm{HBcAg}$ recovered was $49.8 \%$, which corresponds to a purification factor of 2.0. ELISA showed that the $\mathrm{HBcAg}$ recovered is functionally active.
\end{abstract}

Keyword: Anion-exchange expanded-bed adsorption chromatography;(AE-EBAC); Continuous-flow bead milling; Escherichia coli; Hepatitis B core antigen (HBcAg); Hepatitis B virus (HBV); Therapeutic vaccine. 